\title{
Modulated rotating waves in the magnetized spherical Couette system
}

\author{
Ferran Garcia ${ }^{1,2}$, Martin Seilmayer ${ }^{1}$, André Giesecke ${ }^{1}$ \\ Frank Stefani ${ }^{1}$ \\ ${ }^{1}$ Helmholtz-Zentrum Dresden-Rossendorf, Bautzner Landstraße 400, D-01328 \\ Dresden, Germany \\ ${ }^{2}$ Anton Pannekoek Institute for Astronomy, University of Amsterdam, Postbus \\ 94249, 1090 GE Amsterdam, The Netherlands.
}

July 8, 2019

\begin{abstract}
We present a study devoted to a detailed description of modulated rotating waves (MRW) in the magnetized spherical Couette system. The set-up consists of a liquid metal confined between two differentially rotating spheres and subjected to an axially applied magnetic field. When the magnetic field strength is varied, several branches of MRW are obtained by means of three dimensional direct numerical simulations (DNS). The MRW originate from parent branches of rotating waves (RW) and are classified according to Rand's 33. and Coughling \& Marcus 4] theoretical description. We have found relatively large intervals of multistability of MRW at low magnetic field, corresponding to the radial jet instability known from previous studies. However, at larger magnetic field, corresponding to the return flow regime, the stability intervals of MRW are very narrow and thus they are unlikely to be found without detailed knowledge of their bifurcation point. A careful analysis of the spatio-temporal symmetries of the most energetic modes involved in the different classes of MRW will allow in the future a comparison with the HEDGEHOG experiment, a magnetized spherical Couette device hosted at the HelmholtzZentrum Dresden-Rossendorf.
\end{abstract}

Keywords: magnetohydrodynamics - nonlinear waves - bifurcation theory - symmetry breaking - experiments - astrophysics

MSC codes: 37L15 - 37L20 - 65P40 - 76E25 - 76E30 - 85A30

\section{Introduction}

The study of how magnetic fields interact with conducting liquids in rotating spherical containers is crucial for the understanding of many natural phenomena. 
For instance, the Earth's [23] and Solar [34] dynamos are driven by global fluid motions. In addition, the magnetorotational instability (MRI) 2] is considered the best explanation for the transport of angular momentum in accretion disks around black holes and stars, and also in protoplanetary disks [22, allowing matter to fall into the center. Because of its relevance, MRI has been studied experimentally, with GaInSn between two rotating cylinders at HelmholtzZentrum Dresden-Rossendorf (HZDR) [44, 43, 41, and in Maryland [42] with liquid sodium between differentially rotating spheres.

In the context of the Maryland sodium experiment [42, recent numerical [19, 15] and experimental studies [27] have provided alternative interpretations in terms of typical instabilities in magnetized spherical Couette (MSC) flows encouraging further research into this problem. Consider an electrically conducting liquid confined between two differentially rotating spheres and subjected to a magnetic field. Although the spherical Couette (SC) system has a simple formulation it reveals immense complexity even without considering the magnetic field. The problem is described in terms of the three dimensional incompressible Navier-Stokes equations with enforced differential rotation between the boundaries allowing the development of thin shear layers (Stewartson layer [45]) parallel to the rotation axis along the tangent cylinder. Moreover, thin Ekman or Ekman-Hartmann boundary layers [6] appear when the no-slip condition, used to model planetary dynamos and for comparison with laboratory experiments, is imposed at the boundaries. These circumstances make the numerical treatment extremely challenging because of the higher spatial resolution, even in the study of laminar flows at small differential rotation rates.

In case of a resting outer sphere (which is the focus of this paper) three parameters determine the MSC problem: the aspect ratio $\chi=r_{i} / r_{o}$, where $r_{i}$ $\left(r_{o}\right)$ is the radius of the inner (outer) spherical boundary, the Reynolds number Re, measuring the strength of differential rotation, and the Hartmann number Ha measuring the intensity of the applied magnetic field. Without magnetic field the basic axisymmetric SC flow is stable [40] up to a certain critical value of $\mathrm{Re}_{c}$. Beyond this critical point a nonaxisymmetric instability develops and a branch of stable or unstable solutions bifurcates and extends for larger values of Re [30. The flow topology of the instability depends strongly on the gap width. For narrow gaps Taylor-Görtler vortices are formed [52, 51] whereas for sufficiently wide gaps the instability occurs in form of spiral waves [50, 20, 1. With further increasing Re, several bifurcations take place, giving rise to chaotic [50] and eventually turbulent flows. A comprehensive overview of the different flow regimes in the SC system with positive or negative differential rotation was recently given in [49].

In contrast, the impact of magnetic fields on a spherical Couette system has attracted less interest. Although the first instabilities of the basic axisymmetric MSC flow have been characterised in the $(\chi, \mathrm{Re}, \mathrm{Ha})$ parameter space [19, 46], most nonlinear studies briefly explore the parameter space with direct numerical simulations (DNS) and are focused on addressing the influence of input physics such as considering different types of boundary conditions for the magnetic field [21, 15] (insulating or conducting inner sphere allowing magnetic lines 
to pass) or different topologies of the applied magnetic field (dipolar [15, 9], axial [42, 19, 25], or a combination of both [48]). Very recently, an exhaustive numerical study [26, related to the Derviche Torneur sodium experiment in Grenoble [3, revealed several dynamical regimes where coherent structures coexist with turbulent flows when a dipolar magnetic field is imposed.

In case of axially applied magnetic fields and small Re, the studies [21, 19, 46, 25] have shown that the MSC basic state is equatorially symmetric and stable for all Ha. It is described as a strong azimuthal flow associated with a meridional recirculation. At some critical value $\mathrm{Re}_{\mathrm{c}}$, the basic flow becomes unstable to non-axisymmetric perturbations. At low Ha the instability takes the form of an equatorially antisymmetric radial jet instability at the equatorial plane, whereas at large $\mathrm{Ha}$ the instability is equatorially symmetric and related to a shear layer at the tangent cylinder [21, 19. For moderate values of Ha, between the radial jet and the shear layer instability, it takes the form of a meridional return flow instability [19]. We note that the radial jet and return flow instabilities are separated by a Ha interval in which the basic flow stabilises again [19, 46. These instabilities have been recently identified in the HEDGEHOG (ydromagnetic Experiment with Differentially Gyrating sphEres HOlding GaInSn) laboratory experiment [27] at Helmholtz-Zentrum Dresden-Rossendorf when increasing the magnetic field strength (Ha) for a fixed rotation rate (Re).

Both the $\mathrm{SC}$ and the MSC are $\mathbf{S O}(2) \times \mathbf{Z}_{2}$ equivariant systems, i. e., invariant by azimuthal rotations and reflections with respect to the equatorial plane, because of the symmetry of the geometry and the boundary conditions. In $\mathbf{S O}(2)$ symmetric systems, branches of periodic rotating waves (RW) appear after primary Hopf bifurcations [5, 7, 17, when the axisymmetric azimuthal symmetry of the basic state is broken. Secondary Hopf bifurcations [33, 16, 17. give rise to 2-frequency quasiperiodic amplitude or shape modulated rotating waves (MRW) which may have different types of spatio-temporal symmetries. A theoretical characterisation of MRW was first performed in [33] and extended in [16] following several application examples such as the cylindrical TaylorCouette (TC) system. Although the MSC problem is somewhat different to the TC system, it still retains the rotational symmetry, and thus the mathematical analysis of MRW performed in 4], in terms of Floquet theory, also applies. An algorithm for the identification of MRW in $\mathbf{O}(2)$ systems was developed in 32 . in the framework of center bundle construction [28, 16. In case of the MSC problem the existence of RW and MRW has been confirmed by experimental studies [39] and by DNS [21, 19, 15] but their dependence upon parameters, especially the Hartmann number, is still not well understood.

Recently, a continuation method was applied [14] to build up accurate bifurcation diagrams for RW to address the magnetic field strength dependence. The Floquet analysis of periodic orbits was used to determine regions of stability of different azimuthal wave numbers $m$ and to characterise the symmetry of the bifurcated MRW. The present study continues the work of [14 by describing these MRW in terms of the established theory, using the analysis of [33] and [4], and study their dependence upon the magnetic field strength. Our main focus is to determine the dominant azimuthal modes involved in each solution and 
obtain their time scales in correspondence with the azimuthal symmetries of the flow that can be measured in the HEDGEHOG experiment. Our analysis is devoted to a moderate Reynolds number regime and extends previous numerical studies [21, 19, 46, 14] by determining the regions of stability of oscillatory waves and describe them in terms of bifurcation theory. Yet, we leave for future research the study of chaotic attractors that may originate from the flows described in this paper at very weak magnetic fields.

The paper is organized as follows: In $\S 2$ we introduce the formulation of the problem, the numerical method used to integrate the model equations, and some background summarising the theory of MRW classification. In $\S 3$ the bifurcation diagrams as a function of $\mathrm{Ha}$ and the different regions of stability of MRW are presented. The classification of MRW is discussed in $\S 4$ while the analysis of the dominant azimuthal modes is presented in $\S 5$. Finally, in $\S 6$ the paper closes with a summary on the results obtained.

\section{The HEDGEHOG model}

\subsection{The equations and the numerical method}

We consider a conducting fluid of constant density $\rho$, the kinematic viscosity $\nu$, magnetic diffusivity $\eta$ and electrical conductivity $\sigma=1 /\left(\eta \mu_{0}\right)$, where $\mu_{0}$ is the free-space value for the magnetic permeability. The fluid is confined in a spherical shell defined by inner and outer radii $r_{i}$ and $r_{o}$. The outer sphere is at rest while the inner rotates at a constant angular velocity $\Omega$ around the vertical axis $\hat{\mathbf{e}}_{\mathbf{z}}$.

To compare with the HEDGEHOG laboratory experiment 27] the flow is subjected to a uniform axial magnetic field $\mathbf{B}_{\mathbf{0}}=B_{0} \cos (\theta) \hat{\mathbf{e}}_{\mathbf{r}}-B_{0} \sin (\theta) \hat{\mathbf{e}}_{\theta}, \theta$ being the colatitude and $B_{0}$ the magnetic field strength (Fig. 1). To obtain the dimensionless governing equations the length, time, velocity and magnetic field are scaled using the characteristic quantities $d=r_{o}-r_{i}, d^{2} / \nu, r_{i} \Omega$ and $B_{0}$, respectively. We adopt the inductionless approximation $\mathrm{Rm} \ll 1$ valid in the limit of low magnetic Reynolds number $\mathrm{Rm}=\Omega r_{i} d / \eta$. This is reasonable in the case of the HEDGEHOG experiment because the fluid has very low magnetic Prandtl number Pm $=\nu / \eta \sim O\left(10^{-6}\right)$ (eutectic alloy GaInSn [31) and only moderate Reynolds numbers $\operatorname{Re}=\Omega r_{i} d / \nu \sim 10^{3}$ are considered giving rise to $\mathrm{Rm}=\operatorname{PmRe} \sim 10^{-3}$.

The magnetic field is decomposed as $\mathbf{B}=\hat{\mathbf{e}}_{\mathbf{z}}+\mathrm{Rmb}$. By neglecting terms $O(\mathrm{Rm})$, the Navier-Stokes and induction equations become

$$
\begin{gathered}
\partial_{t} \mathbf{v}+\operatorname{Re}(\mathbf{v} \cdot \nabla) \mathbf{v}=-\nabla p+\nabla^{2} \mathbf{v}+\mathrm{Ha}^{2}(\nabla \times \mathbf{b}) \times \hat{\mathbf{e}}_{\mathbf{z}} \\
0=\nabla \times\left(\mathbf{v} \times \hat{\mathbf{e}}_{\mathbf{z}}\right)+\nabla^{2} \mathbf{b} \\
\nabla \cdot \mathbf{v}=0, \quad \nabla \cdot \mathbf{b}=0
\end{gathered}
$$

In this inductionless approximation the MSC system depends upon three nondimensional numbers: the Reynolds number, the Hartmann number and the 


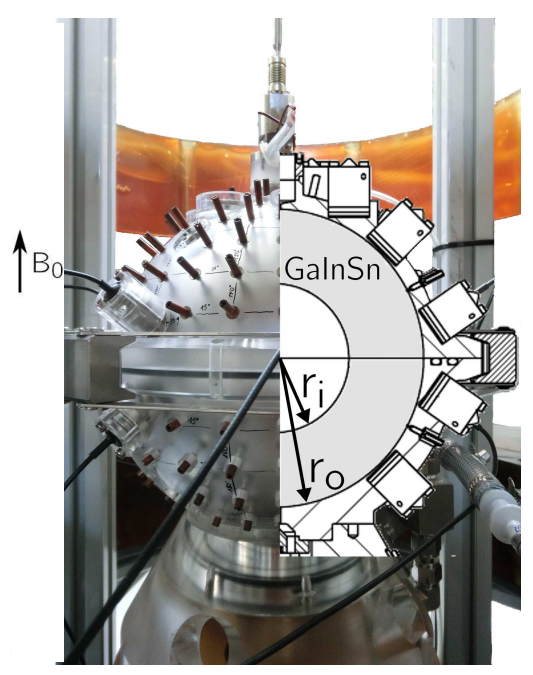

Figure 1: HEDGEHOG experimental configuration. Detail of the rotating spheres showing the ultrasonic Doppler velocimeter (UDV) sensors (thick cylinders) and the electric potential probes (thin needles) attached to the outer sphere.

aspect ratio

$$
\mathrm{Re}=\frac{\Omega r_{i} d}{\nu}, \quad \mathrm{Ha}=\frac{B_{0} d}{\sqrt{\mu_{0} \rho \nu \eta}}=B_{0} d \sqrt{\frac{\sigma}{\rho \nu}}, \quad \chi=\frac{r_{i}}{r_{o}} .
$$

No-slip $\left(v_{r}=v_{\theta}=v_{\varphi}=0\right)$ at $r=r_{o}$ and constant rotation $\left(v_{r}=v_{\theta}=0, v_{\varphi}=\right.$ $\sin \theta)$ at $r=r_{i}$ are the boundary conditions imposed on the velocity field. For the magnetic field, insulating exterior regions are considered in accordance with the experimental setting, see [21] for more details.

The equations are discretized and integrated with the same method as described in 11 and references therein. The velocity field is expressed in terms of toroidal, $\Psi$, and poloidal, $\Phi$, potentials

$$
\mathbf{v}=\nabla \times(\Psi \mathbf{r})+\nabla \times \nabla \times(\Phi \mathbf{r}),
$$

which are expanded in spherical harmonics in the angular coordinates $\left(\mathbf{r}=r \hat{\mathbf{e}}_{\mathbf{r}}\right.$ is the position vector). In the radial direction a collocation method on a GaussLobatto mesh of $N_{r}$ points is used. Specifically, the solution vector $u=(\Psi, \Phi)$ (Eq. 4) is expanded in spherical harmonic series up to degree $L_{\max }$

$$
\begin{aligned}
\Psi(t, r, \theta, \varphi) & =\sum_{l=0}^{L_{\max }} \sum_{m=-l}^{l} \Psi_{l}^{m}(r, t) Y_{l}^{m}(\theta, \varphi), \\
\Phi(t, r, \theta, \varphi) & =\sum_{l=0}^{L_{\max }} \sum_{m=-l}^{l} \Phi_{l}^{m}(r, t) Y_{l}^{m}(\theta, \varphi),
\end{aligned}
$$


with $\Psi_{l}^{-m}=\overline{\Psi_{l}^{m}}, \Phi_{l}^{-m}=\overline{\Phi_{l}^{m}}, \Psi_{0}^{0}=\Phi_{0}^{0}=0$ to uniquely determine the two scalar potentials, and $Y_{l}^{m}(\theta, \varphi)=P_{l}^{m}(\cos \theta) e^{i m \varphi}, P_{l}^{m}$ being the normalized associated Legendre functions of degree $l$ and order $m$. The code is parallelized in the spectral and in the physical space by using OpenMP directives. We use optimized libraries (FFTW3 [10]) for the FFTs in $\varphi$ and matrix-matrix products (dgemm GOTO [18) for the Legendre transforms in $\theta$ when computing the nonlinear terms.

For the time integration, high order implicit-explicit backward differentiation formulas (IMEX-BDF) [11] are used. In the IMEX method we treat the nonlinear terms explicitly in order to avoid solving nonlinear equations at each time step. The Lorenz term is also treated explicitly, which may necessitate a reduced time step in comparison with an implicit treatment. However, this is not a serious issue when moderate Ha are considered, as is the case for the present study. The use of matrix-free Krylov methods (GMRES [36] in our case) for the linear systems facilitates the implementation of a suitable order and time stepsize control for the time integration (see [11 for details on the implementation).

\subsection{Background for Modulated Rotating Waves}

The discretization of the MSC equations Eqs. (1) 3 leads to a system of $n=$ $\left(2 L_{\max }^{2}+4 L_{\max }\right)\left(N_{r}-1\right)$ ordinary differential equations (ODE) of the form

$$
L_{0} \partial_{t} u=L u+B(u, u),
$$

where $L_{0}$ and $L$ are linear operators which include the boundary conditions (see [1] for details). The first operator is invertible and the operator $L$ depends on Ha (the control parameter of the present study) and includes all the linear terms, whereas the non-linear (quadratic) terms are included in the bilinear operator $B$.

The system is $\mathbf{S O}(2) \times \mathbf{Z}_{2}$-equivariant, $\mathbf{S O}(2)$ generated by azimuthal rotations, and $\mathbf{Z}_{2}$ by reflections with respect to the equatorial plane. For fixed Re, at a critical $\mathrm{Ha}_{\mathrm{c}}$ the basic axisymmetric $(m=0)$ flow is unstable to nonaxisymmetric perturbations [19, 46] giving rise to a branch of RWs [7]. These are solutions in which a fixed flow pattern with $m_{1}$-fold azimuthal symmetry is rotating at a frequency $\omega$ in the azimuthal direction [33. For the MSC system, RW have been recently computed [14, as a function of Ha, by means of continuation methods for periodic orbits [38. However, RW can also be computed as fixed points [37, 47] of the MSC system written in a reference frame rotating with the same frequency $\omega$ :

$$
L_{0} \partial_{t} u=L(p) u+B(u, u)+\omega L_{0} \partial_{\varphi} u .
$$

This is because RW satisfy $u(t, r, \theta, \varphi-\omega t)=\tilde{u}(r, \theta, \tilde{\varphi})$, with $\tilde{\varphi}=\varphi-\omega t$. A branch of RW, rotating at a frequency $\omega$ and with $m_{1}$-fold azimuthal symmetry, undergoes secondary Hopf bifurcations at a certain critical value of Ha giving

rise to a branch of MRW. According to 33 these are $\tau$-periodic solutions of 


\section{Visualization of Rand's integers of a MRW}

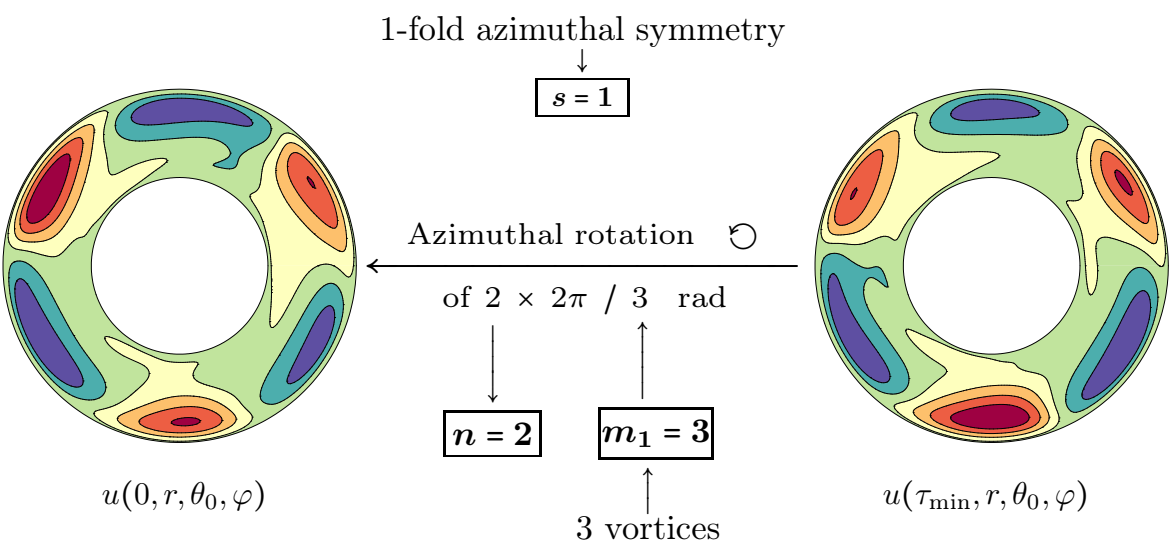

Figure 2: Contour plots of the nonaxisymmetric $(m>0)$ radial velocity on colatitudinal cross-sections at $t=0$ and $t=\tau_{\min }$ for a MRW at $\mathrm{Ha}=3.5$. The main azimuthal drift corresponding to $\omega$ has been removed. The position of colatitudinal cuts is one degree above the equator.

Eq. (8) (i.e. in a reference frame rotating with frequency $\omega$ ) for which there exist a basic (minimal) time $\tau_{\min }>0$ and an integer $0 \leq n<m_{1} / s$ such that

$$
u(t, r, \theta, \varphi)=u\left(t+\tau_{\min }, r, \theta, \varphi+2 \pi n / m_{1}\right) \quad \forall t, \forall \varphi .
$$

The spatio-temporal symmetry of each MRW is then described with three integers $\left(m_{1}, n, s\right)$. They are the $m_{1}$-fold azimuthal symmetry of the parent RW, the integer $n$ related with the minimal period $\tau_{\min }$ in Eq. (9), and the $s$-fold azimuthal symmetry of the MRW (see the scheme in Fig. 2). We note that $s$ can be deduced a priori from the stability analysis (Floquet theory) of a RW close to the bifurcation point [4. In this way, if at the bifurcation point the dominant Floquet multiplier has $m_{2}$-fold azimuthal symmetry then the azimuthal symmetry of the bifurcated MRW will be $s=\operatorname{GCF}\left(m_{1}, m_{2}\right)$ (GCF stands for great common factor). In the original system (Eq. (7)) MRW are quasiperiodic and any frequency obtained from a time spectrum analysis will be a linear combination (with integer coefficients) of Rand's fundamental frequencies 33.

$$
\Omega_{M}=2 \pi / \tau_{\min }, \quad \Omega_{W}=s\left(\omega+n \Omega_{M}\right) / m_{1} .
$$

We note that the MRW period $\tau$ seen when integrating system Eq. (8) (i.e. rotating with frequency $\omega$ ) may not be minimal (this only happens when $n=0$ ). However, there exists a frequency $\omega_{\min }$ and its associated system (Eq. (8)) for which the MRW will exhibit $\tau_{\text {min }}$ periodicity. Detecting this minimal period is important to efficiently obtain MRW with continuation methods for periodic orbits [12, as these methods require a large number of time integrations over one 
modulation period. We note that azimuthally averaged properties of a MRW (either obtained with Eq. (7) or Eq. (8)) will naturally reflect the minimal period as any azimuthal drift is removed [32].

For a MRW close to the bifurcation point its rotating frequency is close to that of the parent RW. An approximation for the second frequency (related with the modulation period) can be obtained by means of Floquet theory in the way we now describe. Let $\mu \in \mathbb{C}$ be a Floquet multiplier and $\lambda \in \mathbb{C}$ be a Floquet exponent 24] of a RW of the MSC system (Eq. 77), rotating at a frequency $\omega$ and with $m_{1}$-fold azimuthal symmetry. The period of the RW is $T=2 \pi / m_{1} \omega$. The Floquet multipliers and exponents are related by $\mu=e^{T \lambda}$. At a Hopf bifurcation point a branch of MRW is born and a modulation period can be approximated by $\tau_{\mathrm{b}}=2 \pi / \Im(\lambda)$. At the bifurcation point $\Re(\lambda)=0$ and thus $T \Im(\lambda)=\operatorname{Arg}(\mu)$ with $\operatorname{Arg}(\mu)$ the complex argument $\left(\mu=|\mu| e^{i \operatorname{Arg}(\mu)}\right)$. Then we have

$$
\tau_{\mathrm{b}}=2 \pi T / \operatorname{Arg}(\mu)=4 \pi^{2} / m_{1} \omega \operatorname{Arg}(\mu) .
$$

We note that $\tau_{\mathrm{b}}$ may neither be the minimal modulation period $\tau_{\min }$ nor the modulation period $\tau$ observed when integrating the system Eq. (8). Nevertheless, $\omega$ and $2 \pi / \tau_{\mathrm{b}}$ are fundamental frequencies as well, so they can be expressed as functions of $\Omega_{M}$ and $\Omega_{W}$ 33. This is useful because Floquet multipliers of RW at bifurcation points have been already obtained in [14] and thus can be used to check the frequencies obtained from a time series analysis of DNS of MRW close to the corresponding bifurcation points.

Besides Rand's classification of MRW [33] the authors of [4 provide an equivalent classification by deriving a functional form for MRW, within the context of Taylor-Couette flows, in terms of bifurcation and Floquet theory. According to [4] a MRW may be described by $\left(m_{1}, c_{1}, m_{2}, c_{2}\right)$ with $m_{1}$ and $c_{1}=\omega$ being the azimuthal symmetry and rotating frequency of the underlying RW, and $m_{2}$ and $c_{2}=c_{1}+\omega_{M} / m_{2}\left(\omega_{M}=2 \pi / \tau\right)$, the azimuthal symmetry and frequency of the Floquet mode, respectively, associated to the modulation (with period $\tau$ ) seen in the frame rotating with frequency $c_{1}$.

\section{Bifurcation diagrams}

For fixed $\chi=0.5$ and in the absence of magnetic field the basic axisymmetric flow becomes unstable at sufficiently large $\operatorname{Re}_{\mathrm{c}} \approx 489$ (see [20]). Then, for $\mathrm{Re}=$ $10^{3}$ the flow is nonaxisymmetric taking the form of a radial jet instability [20. The linear stability analysis of the basic state of [19, 46] showed that with increasing magnetic forcing ( $\mathrm{Ha}$ ) the basic state is recovered at $\mathrm{Ha}_{\mathrm{c}}=12.2$, but loses its stability again at $\mathrm{Ha}_{\mathrm{c}}=25.8$ giving rise to a meridional circulation and a return flow instability. With further increase of Ha the basic state becomes stable again at $\mathrm{Ha}_{\mathrm{c}}=79.4$ taking the form of a strong shear layer parallel to the rotation axis and close to the inner boundary.

At the same $\operatorname{Re}=10^{3}$, branches of unstable/stable rotating waves with azimuthal symmetry $m=2,3,4$ bifurcated from the basic state at the critical points have been recently computed in [14] for the three types of instabilities, 
i.e., for the radial jet instability in the range $\mathrm{Ha} \in[0,12.2]$ and for the return flow and shear layer instabilities in the range $\mathrm{Ha} \in[25.8,79.4]$. The stability analysis (Floquet) of these waves provided the values of the bifurcation points to branches of MRW which are described in this section.

The code and results for the computation of RW and their stability analysis were validated in section 3(c) of [14]. In the present study the same spatial resolutions are employed as the MRW are obtained in the same range of parameters. We use $N_{r}=40$ radial collocation points and a spherical harmonics truncation parameter $L_{\max }=84$ giving rise to a nonlinear system of 563472 degrees of freedom (DOF). When increasing the resolution to $N_{r}=60$ and $L_{\max }=126$ (1903104 DOF), errors below $1 \%$ are obtained (see table 1 of [14]). Numerical experiments with a VSVO time step method (see [11) allows us to determine an appropriate fixed time step (of order $\Delta t=5 \times 10^{-6}$ ) for an accurate fixed time step integration.

We compute MRW using DNS and start each branch with initial conditions built from a RW and its Floquet multiplier close to the bifurcation point. Successive MRW on the branches are obtained from those at slightly different Ha. An alternative procedure allows us to obtain additional branches of MRW without knowing their parent branch of RW (the point where they bifurcate). We set as initial condition a slightly perturbed unstable RW and integrate in time (DNS) until the perturbations are saturated and an attractor is reached. By means of time spectrum analysis we infer the quasiperiodic character of the DNS. By imposing azimuthal symmetry constraints on the DNS (by retaining only certain modes on the spherical harmonic expansion) we are able to capture unstable MRW.

Figure 3(a,b) displays the time average and minimum/maximum values of the volume averaged nonaxisymmetric $(m>0)$ kinetic energy $K_{n a}$ versus the Hartmann number in the low magnetic field regime corresponding to the radial jet instability. Branches for RW with $m$-fold azimuthal symmetry $m=2,3,4$ were obtained in [14, where several bifurcation points along the branches were computed as well. This information is summarised in Table 1. A branch of RW or MRW is denoted by $\mathrm{RW}_{s}^{m_{\max }}$ or $\mathrm{MRW}_{s}^{m_{\max }}$ when the waves belonging to the branch have $s$-fold azimuthal symmetry and their most energetic azimuthal wave number (with the exception of $m=0$ ) is $m_{\max }$. For instance, a wave in the $\mathrm{MRW}_{1}^{3}$ branch has 1 -fold azimuthal symmetry (i. e. invariant by $2 \pi$ azimuthal rotations) and $K_{3} \geq K_{m}(m \neq 0), K_{m}$ being the mean rms kinetic energy contained in the azimuthal wave number $m$.

The branches of $\mathrm{RW}_{2}^{2}$ and $\mathrm{RW}_{4}^{4}$ are unstable and thus the bifurcated $\mathrm{MRW}_{2}^{2}$ (at $\mathrm{Ha}_{\mathrm{c}}=5.27$ ) and $\mathrm{MRW}_{2}^{4}$ (at $\mathrm{Ha}_{\mathrm{c}}=9.98$ ) are unstable as well. Because of the $m=2$ azimuthal symmetry of the corresponding Floquet mode at the bifurcation, the branches of $\mathrm{MRW}_{2}^{2}$ and $\mathrm{MRW}_{2}^{4}$ can be computed with $m=2$ azimuthal symmetry constraints imposed on the DNS. In contrast to $\mathrm{RW}_{2}^{2}$ or $\mathrm{RW}_{4}^{4}$, the branch of $\mathrm{RW}_{3}^{3}$ has a stable Ha interval. The $\mathrm{RW}_{3}^{3}$ branch corresponds to the branch of the most unstable linear mode of the basic state [46], emerging at $\mathrm{Ha}_{\mathrm{c}}=12.2$. At $\mathrm{Ha}_{\mathrm{c}}=3.95, \mathrm{RW}_{3}^{3}$ becomes unstable by decreasing $\mathrm{Ha}$, at a Hopf bifurcation, giving rise to a branch of stable $\mathrm{MRW}_{1}^{3}$. By further decrease 

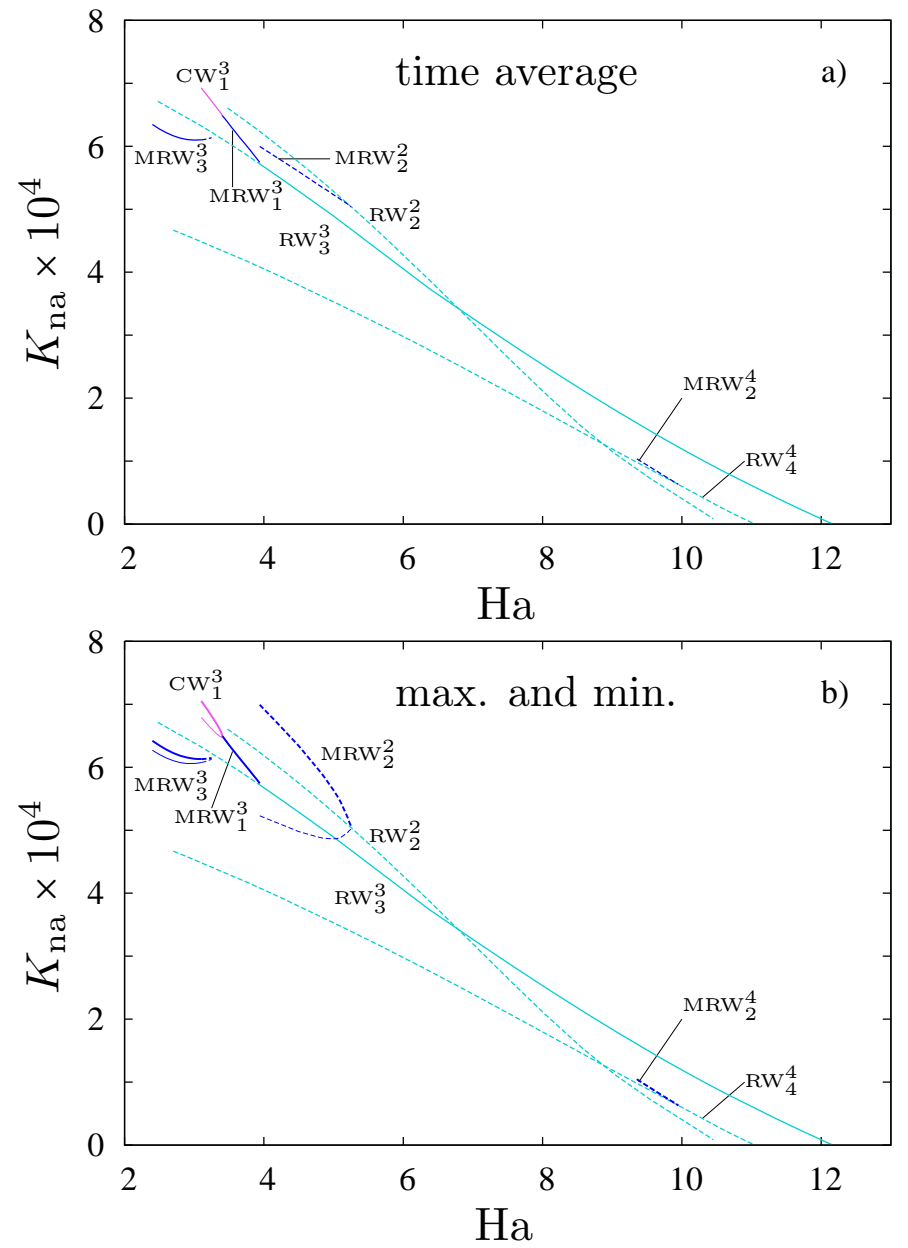

Figure 3: Bifurcation diagrams for rotating and modulated rotating waves (RW/MRW) corresponding to the equatorially asymmetric radial jet instability. The label notation is RW/MRW $m_{\text {max }}^{m_{\max }}, s$ being the azimuthal symmetry of the waves and $m_{\max } \neq 0$ their most energetic azimuthal wave number. A stable branch of complex waves with 3 frequencies, $m_{\max }=3$, and azimuthal symmetry $s=1$ is also included $\left(\mathrm{CW}_{1}^{3}\right)$. For $\mathrm{Ha} \rightarrow 0$ different complex and even chaotic flows are identified but their description is out of scope of the present study. The volume averaged nonaxisymmetric $(m>0)$ kinetic energy $K_{\text {na }}$ is plotted versus the Hartmann number Ha. (a) Time average and (b) maximum (thick line) and minimum (thin line) of the time series. Solid/dashed lines mean stable/unstable waves. 
of Ha (along the $\mathrm{RW}_{3}^{3}$ branch) a second Hopf bifurcation, which does not break the $m=3$ azimuthal symmetry, gives rise to a branch of unstable $\mathrm{MRW}_{3}^{3}$ at $\mathrm{Ha}_{\mathrm{c}}=3.33$. Again these waves can be computed with azimuthal symmetry constraints as done for the case of $\mathrm{MRW}_{2}^{2}$ or $\mathrm{MRW}_{2}^{4}$. At $\mathrm{Ha}_{\mathrm{c}} \approx 3.1$ the branch of $\mathrm{MRW}_{3}^{3}$ becomes stable to arbitrary random perturbations with $m=1$-fold azimuthal symmetry.

Table 1: Critical parameters of the equatorially asymmetric RWs at the bifurcations where they change the stability $(|\mu|=1)$. They are obtained by inverse interpolation with a polynomial of degree 5 . The sign of the real part of the Floquet exponent of the two leading eigenfunctions before and after the transition is shown. The azimuthal symmetry $\left(2 \pi / m_{2}\right.$ azimuthal periodicity) of the eigenfunction with a change of sign is also stated. We use $\mathrm{ES}=1$ for equatorially symmetric flows, $\mathrm{ES}=0$ otherwise. The rotating frequency $\omega$ and the fundamental frequency of the time spectrum $f_{\omega}=m \omega / 2 \pi$, the argument of the Floquet multiplier $\operatorname{Arg}(\mu)$ and the corresponding modulation period $\tau_{\mathrm{b}}$ and fundamental frequency $f_{\mathrm{b}}=1 / \tau_{\mathrm{b}}$ (see Eq. (11)), are also tabulated.

\begin{tabular}{llllllllll}
\hline$m$ & ES & Signs at transition & $\mathrm{Ha}_{\mathrm{c}}$ & $m_{2}$ & $\omega$ & $f_{\omega}$ & $\operatorname{Arg}(\mu)$ & $\tau_{\mathrm{b}}$ & $f_{\mathrm{b}}$ \\
\hline 2 & 0 & $(+,+) \rightarrow(+,-)$ & 5.27 & 2 & 132.40 & 42.145 & 0.291 & 0.512 & 1.952 \\
3 & 0 & $(+,+) \rightarrow(+,-)$ & 3.33 & 3 & 138.88 & 66.310 & 2.131 & 0.044 & 22.492 \\
3 & 0 & $(+,-) \rightarrow(-,-)$ & 3.95 & 1 & 139.07 & 66.399 & 0.774 & 0.122 & 8.181 \\
4 & 0 & $(+,+) \rightarrow(+,-)$ & 9.98 & 2 & 136.08 & 86.630 & 3.131 & 0.023 & 43.173 \\
\hline 2 & 1 & $(+,+) \rightarrow(+,-)$ & 29.98 & 2 & 56.44 & 17.966 & 0.632 & 0.553 & 1.807 \\
4 & 1 & $(-,-) \rightarrow(+,-)$ & 31.95 & 1 & 101.62 & 64.691 & 2.076 & 0.047 & 21.377 \\
\hline
\end{tabular}

By taking initial conditions on the $\mathrm{MRW}_{1}^{3}$ branch, a stable branch of quasiperiodic waves, with three frequencies and with $m_{\max }=3$ and azimuthal symmetry $m=1$ (labelled $\mathrm{CW}_{1}^{3}$ ), is found if $\mathrm{Ha}$ is decreased beyond the threshold of $\mathrm{Ha}_{\mathrm{c}}=3.4$. This sequence of bifurcations -basic steady state, $\mathrm{RW}_{3}^{3}$, quasiperiodic with two frequencies $\mathrm{MRW}_{1}^{3}$ and quasiperiodic with three frequencies $\mathrm{MRW}_{1}^{3}$ - seen at low Ha for the radial jet instability with $m_{\max }=3$ corresponds to the Ruelle-Takens scenario [35, 8, which is typical for systems with symmetry [33, 16. Figure 4(a) displays Poincaré sections (see figure caption) for a selection of four different flows which evidence the sequence of bifurcations. At $\mathrm{Ha}=4$ the Poincare section of a $\mathrm{RW}_{3}^{3}$ (periodic orbit) is a point while at the lower values $\mathrm{Ha}=3.8$ (red curve) and $\mathrm{Ha}=3.5$ (blue curve) the sections of $\mathrm{MRW}_{1}^{3}$ (invariant tori) are closed curves. At $\mathrm{Ha}=3.1$ the cloudy Poincaré section exhibits features of a 3 frequency solution. We note that an accurate computation of bifurcation points to MRW with 3 frequencies in $\mathbf{S O}(2)$ systems could be done efficiently as in 12 by considering MRW as periodic orbits.

An interesting property of $\mathrm{MRW}_{1}^{3}$, but also of $\mathrm{MRW}_{2}^{4}$, is that the amplitude of oscillations of azimuthally averaged properties ( $K_{n a}$ in particular) is very small. In fact, the maximum and minimum curves of Fig. 3(b) are indistinguishable from each other. This may lead to confuse $\mathrm{MRW}_{1}^{3}$ or $\mathrm{MRW}_{2}^{4}$ with RW as their azimuthally averaged properties appear to be nearly constant. This is 

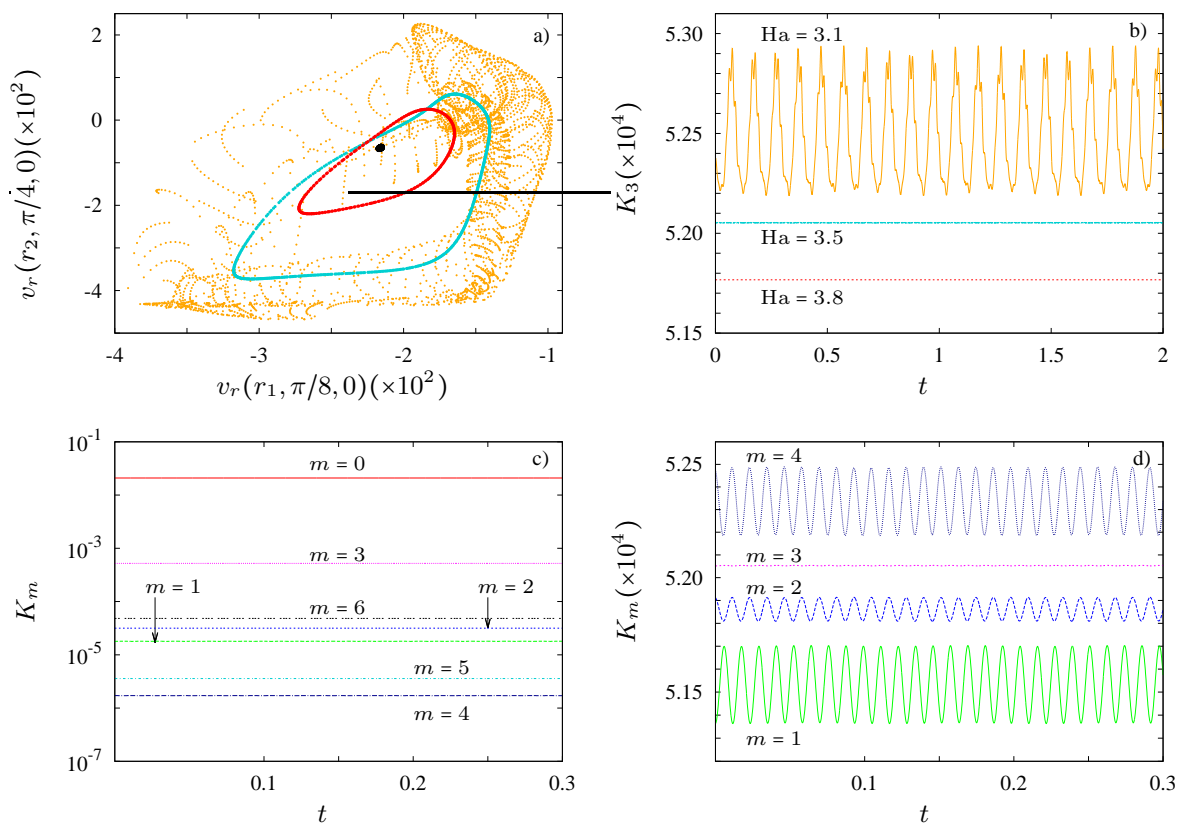

Figure 4: (a) Poincaré sections defined by $v_{r}\left(r_{2}, \pi / 8,0\right)=-6 \times 10^{-2}$ for a $\mathrm{RW}_{3}^{3}$ at $\mathrm{Ha}=4$ (black point) for $\mathrm{MRW}_{1}^{3}$ at $\mathrm{Ha}=3.8,3.5$, from smaller (red) to larger (blue) closed curves, and a $\mathrm{CW}_{1}^{3}$ at $\mathrm{Ha}=3.1$ (cloud of orange dots). The radial positions are $r_{1}=r_{i}+0.15 d$ and $r_{2}=r_{i}+0.5 d$. (b) The oscillations of the $m=3$ component of the flow are noticeable from the 3rd instability (the onset of 3rd frequency via Hopf bifurcation). From top to bottom $K_{3}$ for $\mathrm{Ha}=3.1$ (orange), $\mathrm{Ha}=3.5$ (blue), and $\mathrm{Ha}=3.8$ (red). (c) Time series of kinetic energy densities $K_{m}$ for a MRW ${ }_{1}^{3}$ at $\mathrm{Ha}=3.5$. (d) Detail of (c) with scaled $K_{m}$ to stress the small relative variance of the oscillations. The scaling factors are 28.7, 16.4, 1, 306 for $m=1,2,3,4$, respectively. 

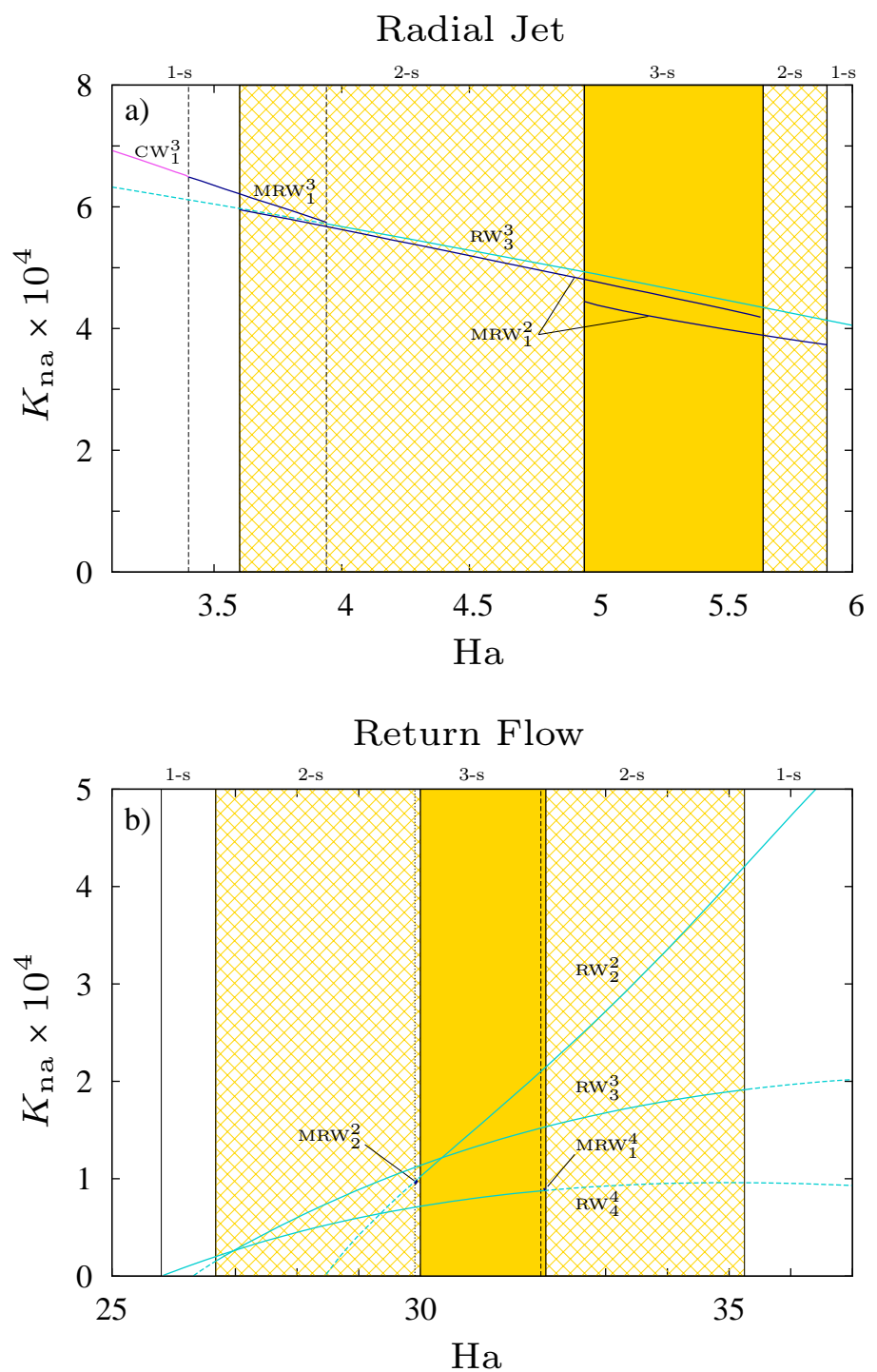

Figure 5: Bifurcation diagrams of the volume averaged nonaxisymmetric $(m>$ 0 ) kinetic energy $K_{\text {na }}$ versus the Hartmann number Ha. (a) equatorially asymmetric radial jet instability and (b) equatorially symmetric return flow instability. Notation is as in Fig. 3. The different regions of multistability are highlighted with bands: one stable solution (empty), bi-stability (cross-hatched) and tri-stability (solid). Vertical dashed lines separate different type of solutions in each band. In contrast to the radial jet instability, less different types of modulated rotating waves, on very narrow regions, have been found for the return flow instability (panel (b)). Only $\mathrm{MRW}_{1}^{4}$ are stable. The dotted vertical line delimit the thin band where unstable $\mathrm{MRW}_{2}^{2}$ are found. 
illustrated in Fig. 4(b) displaying the kinetic energy contained in the $m=3$ azimuthal wave number $\left(K_{3}\right)$ for three different flows. For solutions lying on the $\mathrm{MRW}_{1}^{3}$ branch $(\mathrm{Ha}=3.5,3.8)$ the kinetic energy $K_{3}$ seems to be constant contrasting to the oscillatory behaviour seen at $\mathrm{Ha}=3.1$. The amplitudes of oscillation in the kinetic energy wave number $m$ spectra for the $\mathrm{MRW}_{1}^{3}$ at $\mathrm{Ha}=3.5$ shown in Fig. 4 (c) are extremely small and become only visible when the time series of $K_{m}$ are examined in detail (Fig. $4(\mathrm{~d})$ ).

Aside the branches of MRW shown in Fig. 3 and born at the bifurcation points summarised in Table 1, we have found two additional branches with $m=1$-fold azimuthal symmetry and $m_{\max }=2$ labelled as $\mathrm{MRW}_{1}^{2}$. These MRW correspond to the radial jet instability and display noticeable oscillations of the azimuthally averaged physical properties. They are stable along all the branch as no azimuthal constraint is imposed on the DNS. The branches of $\mathrm{MRW}_{1}^{2}$ are displayed in Fig. 5(a) together with the branches of $\mathrm{RW}_{3}^{3}, \mathrm{MRW}_{1}^{3}$ and $\mathrm{CW}_{1}^{3}$ already shown in Fig. 3 (a). The intervals of stability of the different waves overlap, giving rise to regions of multistability of two and even three different types of waves. In the limits of the 3-stability region we have found hysteretic behaviour between the two branches of $\mathrm{MRW}_{1}^{2}$. Because we are using DNS the branches of $\mathrm{MRW}_{1}^{2}$ are lost close to $\mathrm{Ha}=3.5$ (or $\mathrm{Ha}=6$ ) as they become unstable and all the azimuthal symmetries are broken. The use of continuation methods [38, 12] may help to understand their origin.

According to Fig. 5(a) for the radial jet instability (at low Ha) oscillatory solutions (MRW and 3 frequency waves) are stable in a relatively wide interval of Hartmann numbers. This contrasts with the dynamical behaviour obtained in the return flow regime (moderate $\mathrm{Ha}$ ) for which very narrow intervals of stability of only two types of MRW are found (see Fig. 5(b) and Table 1). By increasing Ha further, only RW are found for the case of the shear layer instability [14] at the selected $\operatorname{Re}=10^{3}$ of the present study. According to these results, increasing magnetic field results in a decrease of the flow complexity in the sense that less different types of waves are obtained and the flow solutions exhibit a simpler time dependence. For example, for Ha $\in[35.24,79.42]$ only RW with $m=2$-fold azimuthal symmetry are found [14.

\section{Classification of MRW}

Several examples of MRW (belonging to each of the branches previously described) are classified in this section following Rand's 33 and Coughling \& Marcus 4 theoretical work, previously summarised in Sec. 2.2. The numerical approach for the classification and the flow patterns of the MRW are described as well.

\subsection{Classification algorithm}

Our procedure is based on DNS (after filtering the initial transient) of the MSC system equations (Eq. (7)) as well as on a rotating frame with selected rotating 
frequency (Eq. (8)) in the way we now describe:

1 Given an initial condition a time evolution of system Eq. (7) is performed to determine $f_{\omega}$. The latter corresponds to the main peak of the frequency spectrum and can be found accurately by using Laskar's algorithm [29] applied to a time series of the radial velocity component $v_{r}(t, p)$ picked up at a point inside the shell $p=(r, \theta, \varphi)=\left(\left(r_{i}+r_{o}\right) / 2, \pi / 4,0\right)$. The azimuthal symmetry $s$ of the MRW and the azimuthal symmetry $m_{1}$ of the underlying rotating wave are detected by computing the azimuthal wave number's kinetic energy spectra. We have found in all cases that $m_{1}=m_{\max }$ corresponds to the wave number of maximum amplitude (except $m=0$ ). The rotating frequency of the MRW is $\omega=2 \pi f_{\omega} / m_{1}$.

2 Once $\omega$ is known, we integrate system Eq. (8) approximately one modulation period $\tau$ (by imposing $\left|v_{r}(0, p)-v_{r}(\tau, p)\right| / v_{r}(0, p)<10^{-3}$ ) and capture the flow patterns at times $t=0, \tau / 4, \tau / 3, \tau / 2$. If $\tau$ is not the minimal period then the patterns at time $t=0$ and at time $t=\tau / j$, for some $1<j \leq m_{1}$, should differ by an azimuthal rotation of $2 \pi n / m_{1}$ degrees. Then, the minimal rotation period $\tau_{\min }$ and the integer $n$ appearing in the labels $\left(m_{1}, n, s\right)$ of Rand's classification [33] are identified. Fundamental Rand's frequencies are easily obtained from Eq. 10 .

3 Aside $c_{1}=\omega$ and $m_{1}$ the classification of 4 involves the integer $m_{2}$ and frequency $c_{2}=c_{1}+\omega_{M} / m_{2}$ with $\omega_{M}=2 \pi / \tau$. The integer $m_{2}$ corresponds to the azimuthal symmetry of the underlying Floquet mode which can be inferred from the DNS by removing the multiples of $m_{1}$ in the spherical harmonics expansion.

Rand's 33 parameters correspond to the integers $m_{1}, n$ and $s$ (see Eq. 9p) and the frequencies $f_{\omega}=m_{1} \omega / 2 \pi, f_{M}=1 / \tau_{\min }$ and $f_{W}=s\left(f_{\omega} / m_{1}+n f_{M}\right) / m_{1}$ (from Eq. 10) whereas Coughling \& Marcus [4 parameters are the integers $m_{1}$ and $m_{2}$ and the frequencies $f_{\omega}$ and $f_{2}=m_{2} f_{\omega} / m_{1}+f_{\tau}$. The results of the classification are summarised in Table 2 for the 6 different types of MRW found at low magnetic forcing corresponding to the radial jet instability.

We note that with the exception of the $\mathrm{MRW}_{1}^{2}$, the parent branch of RW and the corresponding Floquet mode at the bifurcation is known [14 and thus can be used to verify the results obtained from DNS. For $\mathrm{MRW}_{1}^{4}$ and $\mathrm{MRW}_{2}^{4}$ the Laskar frequency closest to that obtained theoretically at the bifurcation point (see Table 1 and Sec. 2.2 is $f_{\mathrm{b}}=m_{2} f_{\omega} / m_{1}-f_{\tau}$, with $f_{\tau}=1 / \tau$. In contrast, for the $\mathrm{MRW}_{2}^{2}$ and $\mathrm{MRW}_{3}^{3}$ we obtain $f_{\mathrm{b}}=f_{\tau}$. For the $\mathrm{MRW}_{1}^{2}$ at $\mathrm{Ha}=5$, the origin of which (bifurcation point) is unknown, we assume $f_{\mathrm{b}}=m_{2} f_{\omega} / m_{1}-f_{\tau}$ as in the case of MRW with $m_{2} \neq m_{1}$.

\subsection{Flow patterns for the MRW}

Figure 6 visualises the flow patterns and the spatio-temporal symmetries for three examples of MRW. Specifically, the nonaxisymmetric radial velocity is 

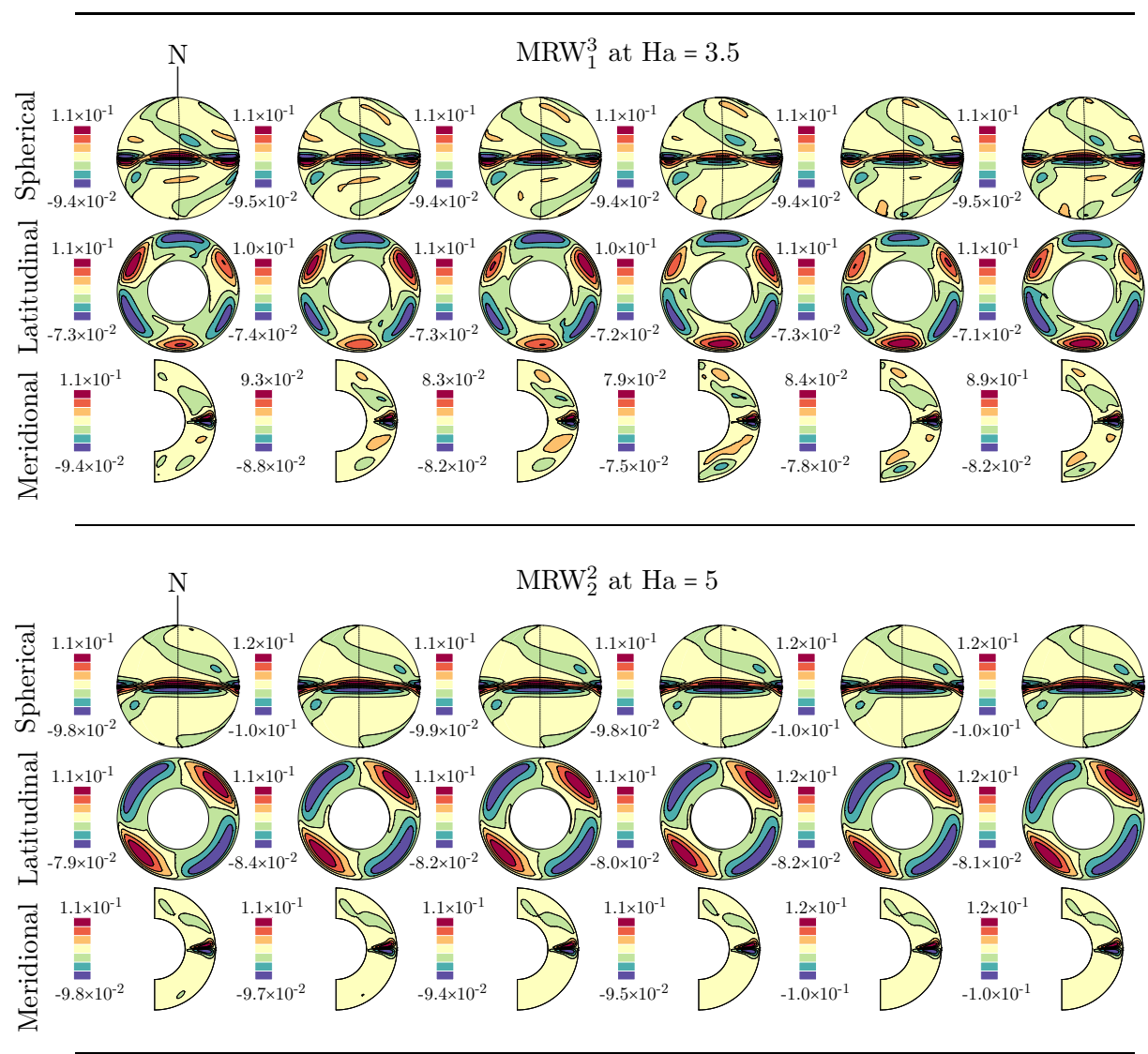

$\mathrm{MRW}_{1}^{2}$ branch 1 at $\mathrm{Ha}=5$

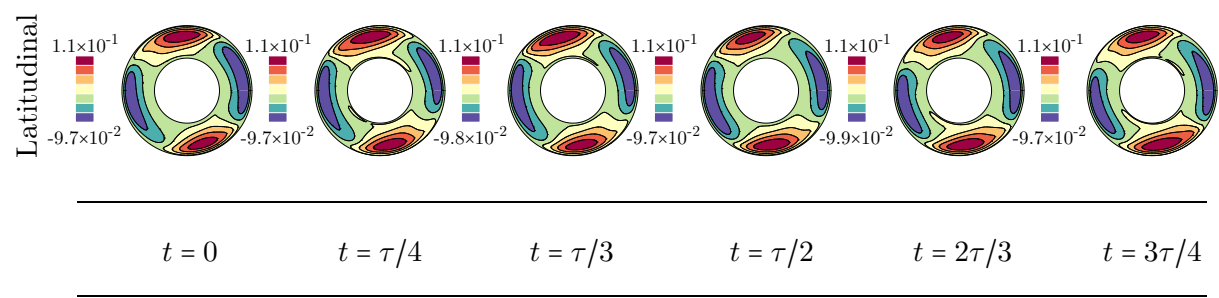

Figure 6: Contour plots of the nonaxisymmetric $(m>0)$ radial velocity on spherical, colatitudinal and meridional cross-sections (from 1st to 3rd row) over a period $\tau$ for the $\mathrm{MRW}_{1}^{3}$ at $\mathrm{Ha}=3.5$. The same sections for the $\mathrm{MRW}_{2}^{2}$ at $\mathrm{Ha}=5$ are from 4 th to 6 th rows. Last row correspond to the colatitude section of the $\mathrm{MRW}_{1}^{2}$ lying on branch 1 at $\mathrm{Ha}=5$. The main azimuthal drift corresponding to $\omega$ has been removed. The cross-sections at $t=0$ are taken through a maximum of $v_{r}$. The position of colatitudinal and meridional cuts are marked with a vertical line on the spherical sections which are displayed from a $0^{\circ}$ latidude point of view. 
Table 2: Rand's classification 33 for the modulated rotating waves: the integers $m_{1}, n$ and $s$ and the frequencies $f_{\omega}=m_{1} \omega / 2 \pi, f_{M}=1 / \tau_{\min }, f_{W}=s\left(f_{\omega} / m_{1}+\right.$ $\left.n f_{M}\right) / m_{1}, f_{\tau}=1 / \tau$ and $f_{\omega_{\min }}=m_{1} \omega_{\min } / 2 \pi$. The modulation period $\tau$ is that exhibited by any scalar field, at a point inside the shell, when integrating the system rotating at a frequency $\omega$ (i.e. removing the main azimuthal drift). The modulation period $\tau_{\min }$ corresponds to that of any azimuthally averaged property and that exhibited by any scalar field, at a point inside the shell, when integrating the system rotating at a frequency $\omega_{\min }$. If $\tau=k \tau_{\min }$ for any integer $k>1$ then $\omega_{\min }=\omega-2 \pi / \tau$. The classification of [4 involves $m_{1}$ and $f_{\omega}$ but also $m_{2}$ (the azimuthal symmetry of the Floquet multiplier) and $f_{2}=m_{2} f_{\omega} / m_{1}+f_{\tau}$. For the $\mathrm{MRW}_{1}^{2}$ at $\mathrm{Ha}=5$, (1) stands for branch 1 and (2) for branch 2.

\begin{tabular}{llllllllllll}
\hline $\mathrm{Ha}$ & $m_{1}$ & $n$ & $s$ & $f_{\omega}$ & $f_{M}$ & $f_{W}$ & $f_{\tau}$ & $f_{\omega_{\min }}$ & $f_{\mathrm{b}}$ & $m_{2}$ & $f_{2}$ \\
\hline 5.00 & 2 & 0 & 2 & 42.12 & 2.12 & 21.06 & 2.12 & 42.12 & 2.12 & 2 & 44.24 \\
$5.00^{(1)}$ & 2 & 1 & 1 & 42.19 & 4.19 & 12.64 & 2.10 & 38.00 & 19.00 & 1 & 23.89 \\
$5.00^{(2)}$ & 2 & 1 & 1 & 42.06 & 4.45 & 12.74 & 2.28 & 37.50 & 18.75 & 1 & 23.31 \\
3.5 & 3 & 2 & 1 & 66.05 & 42.86 & 35.91 & 14.29 & 23.19 & 7.73 & 1 & 36.30 \\
3.25 & 3 & 0 & 3 & 66.32 & 22.52 & 22.11 & 22.52 & 66.32 & 22.52 & 3 & 88.85 \\
9.8 & 4 & 1 & 2 & 86.58 & 0.26 & 10.95 & 0.13 & 86.07 & 43.16 & 2 & 43.42 \\
\hline
\end{tabular}

displayed at time instants $t=0, \tau / 4, \tau / 3, \tau / 2,2 \tau / 3,3 \tau / 4$ to capture the evolution over a modulation period $\tau$. The main azimuthal drift corresponding to $\omega$ has been removed by integrating the system of Eq. (8). Spherical, colatitudinal and meridional cross-sections through a relative maximum of $v_{r}$ provide a global view of the flow structure. Supplementary movies displaying the time evolution over a modulation period, in the frame rotating with the corresponding frequency (Eq. (8)), are provided for the colatitudinal sections of each MRW (2nd, 4rth and 7th rows) in Fig. 6. MRW are strongly equatorially asymmetric in the form of a radial jet near the equatorial plane as described for RW corresponding to the radial jet instability [14. However, for MRW fluid motions start to be noticeable within the cylinder parallel to the rotation axis and tangent to the inner sphere (see meridional sections). For the $\mathrm{MRW}_{1}^{3}$ at $\mathrm{Ha}=3.5$ the patterns at $t=0$ and $t=\tau / 3$ are the same but differ by an azimuthal rotation of $4 \pi / 3$ (see colatitudinal sections). Then, $\tau / 3$ is the minimal period and the MRW is of class $(3,2,1)$ according to Rand's description 33 . For the $\mathrm{MRW}_{2}^{2}$ at $\mathrm{Ha}=5$ the patterns at all time instants are different and thus $\tau$ is the minimal period and the MRW is of class $(2,0,2)$. Finally, for the $\mathrm{MRW}_{1}^{2}$ at $\mathrm{Ha}=5$ the patterns at $t=0$ and $t=\tau / 2$ are the same but differ by an azimuthal rotation of $\pi$. Then, $\tau / 2$ is the minimal period and the MRW is of class $(2,1,1)$. 


\section{Dominant azimuthal mode analysis}

This section is devoted to a detailed description of the main azimuthal modes involved in four examples of MRW. They are $\mathrm{MRW}_{1}^{3}$ at $\mathrm{Ha}=3.5, \mathrm{MRW}_{2}^{2}$ at $\mathrm{Ha}=5$, and two $\mathrm{MRW}_{1}^{2}$ on branch 1 and 2 , both at $\mathrm{Ha}=5$. The later choice makes sense as experimental data at $\mathrm{Ha}=5$ is already available [27. For each example of MRW we select three sets of positive azimuthal wave numbers in its spherical harmonics expansion (Eqs. (5.60) containing more than $96 \%$ of the nonaxisymmetric $(m>0)$ kinetic energy. Apart from being the most energetic modes, the choice of the sets is also motivated to reflect the azimuthal symmetry of the underlying RW and that of the perturbation (Floquet mode) giving rise to the modulation. Indeed, the MRW can then be reasonably approximated by the sum of the three sets of modes rather than by the full expansion of Eqs. (5 6). From a time integration of a MRW, with the main azimuthal drift removed (i.e. integrating the system Eq. (8)), we can follow individually the evolution of each set (the sum of their modes) over a modulation period $\tau$. This provides useful information about the location of maximum flow velocities for specific azimuthal symmetries and hence is important from the experimental point of view, as flow measurement probes in the HEDGEHOG experiment can be located adequately to capture certain azimuthal symmetries.

For each set of modes we consider individually their equatorially symmetric as well as antisymmetric component to study in detail the equatorial symmetry. MRW are dominated by the antisymmetric contribution as is characteristic for radial jet instabilities at low magnetic field [19. Measurement probes located symmetrically with respect to the equator help to infer the equatorially symmetric (ES) as well as antisymmetric (EA) part of the flow in experimental devices [27]. In addition, any measurement in the equatorial plane refers only to the ES part of the flow (as the EA part of any field is zero at the equator). The dominant azimuthal wave number of the ES flow $m_{\text {max }}^{\text {s }}$ may differ from the dominant azimuthal wave number $\left(m_{\max }\right)$ of the total solution which, indeed is the case for our examples. We have found $m_{\max }^{\mathrm{s}}=2 m_{\max }$ for all the classes of MRW. Then, the time series of a scalar field at a point $(r, \pi / 2, \varphi)$ (equator) will have half the period than that at a point $(r, \theta, \varphi), \theta \neq \pi / 2$ (off the equator), which is verified in our simulations.

\section{1 $\mathrm{MRW}_{1}^{3}$ at $\mathrm{Ha}=3.5$}

For the $\mathrm{MRW}_{1}^{3}$ at $\mathrm{Ha}=3.5$ we consider three set of modes $m=1, m=2$ and $m=3 k, k \in \mathbb{Z}$ (containing all the modes which are multiples of 3 ). Their kinetic energy content is $K_{m=1} / K_{m>0}=0.03, K_{m=2} / K_{m>0}=0.05$ and $K_{m=3 k} / K_{m>0}=0.91$, where $K_{m}$ is the time and volume averaged kinetic energy contained in each set of modes and $K_{m>0}$ is the nonaxisymmetric kinetic energy. Figure 7 displays a snapshot of the nonaxisymmetric radial velocity in spherical, colatitudinal and meridional cross-sections similar to Fig. 6. Each column represents the ES or EA part of each set of modes extracted from the spherical harmonics expansion of the total flow. Supplementary movies display- 

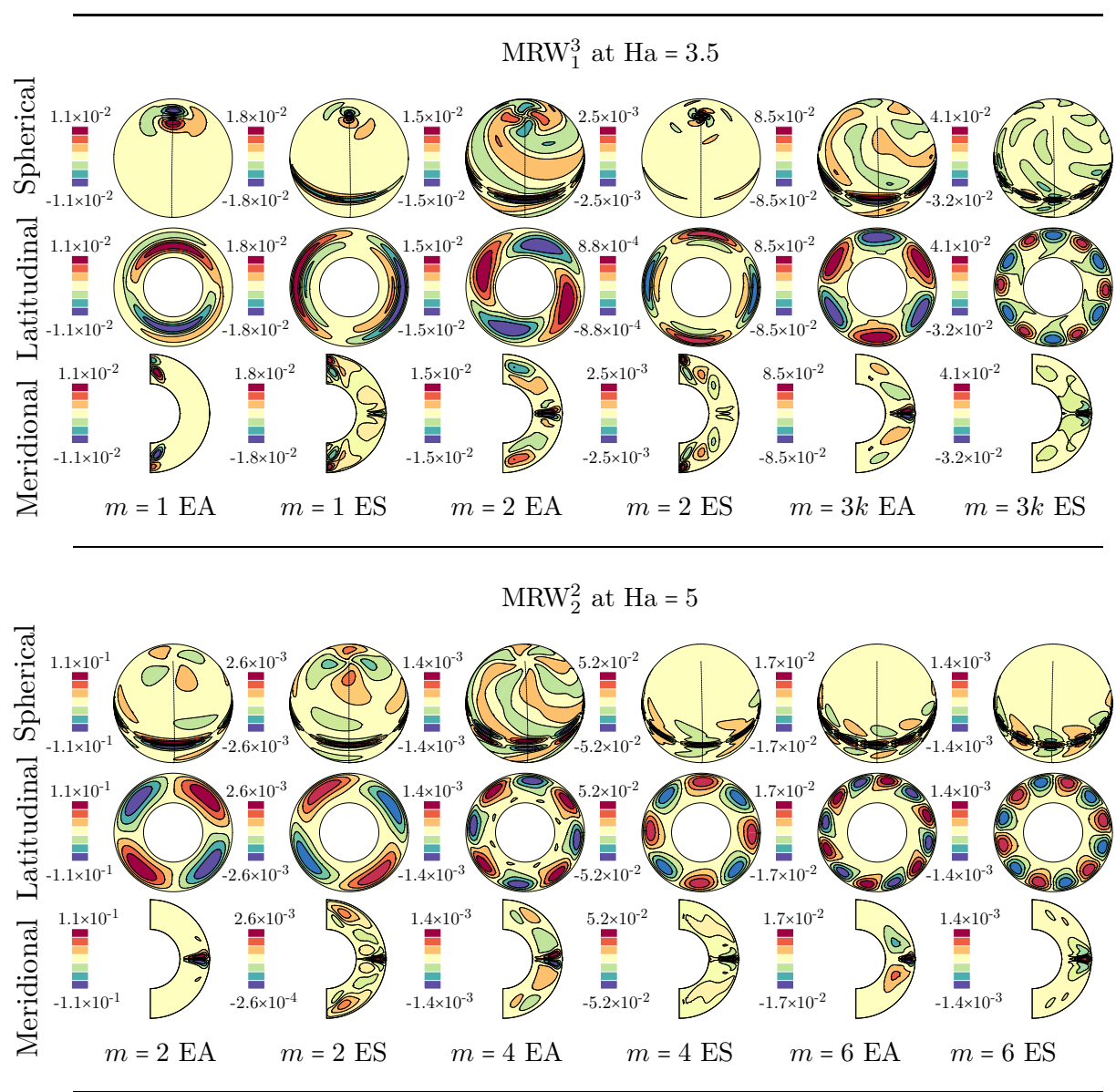

Figure 7: Most energetic modes with $m=1, m=2$ and $m=3 k k \in \mathbb{Z}$, equatorially antisymmetric (EA) or symmetric modes (ES) of the $\mathrm{MRW}_{1}^{3}$ at $\mathrm{Ha}=3.5$ (top 3 rows) and modes $m=2,4,6$ of the $\mathrm{MRW}_{2}^{2}$ at $\mathrm{Ha}=5$ (bottom 3 rows). Cross-sections are as Fig. 6 but the spherical section point of view is at $45^{\circ}$ latitude. 
ing the time evolution over a modulation period, in the frame rotating with the corresponding frequency (Eq. (8)), are provided for each colatitudinal section in 2nd and 5th rows of Fig. 7. We recall that the corresponding time evolution of the nonaxisymmetric part of the flow (also provided as supplementary movie) will be accurately approximated by the sum of the flow components shown in Fig. 7. The inspection of the latter figure and its associated movies provide relevant information summarised in the following (also in Table 3):

- The set $m=3 k$ is clearly associated with the underlying RW. The EA part (5th column of Fig. 7) of this set of modes is dominant as the maximum radial velocity is more than twice that of the ES part (6th column of Fig. 7). In addition, the EA part is steady as is the case of a RW. The ES part is not azimuthally drifting, but changing its shape with a period $\tau / 2$ associated to the modulation. The azimuthal symmetry of the EA $(m=3)$ and $\operatorname{ES~}(m=6)$ part is different (see colatitude sections) and thus measured velocities for the EA and ES part exhibit different periods (differing by a factor of two).

- The set $m=1$ only contains a single azimuthal wave number and is related with the Floquet multiplier giving rise to this branch of MRW. The radial velocity patterns (1st and 2nd columns) resemble quite well those of the Floquet multiplier given in Fig. 7 of [14] and evidence that polar fluid motions appear noticeable for the $\mathrm{MRW}_{1}^{3}$ but are very weak for the $\mathrm{RW}_{3}^{3}$ (compare meridional sections of 1 st/2nd columns with 5 th/6th columns). The time evolution of the $m=1 \mathrm{EA}$ part corresponds to a polar vortex with a purely retrograde azimuthal rotation (i. e. the pattern remains the same) of period $\tau / 2$ whilst in the ES case, apart from the azimuthal drift (in the prograde way), there is a very weak change of pattern with period $\tau$.

- The flow patterns of the single mode set $m=2$ are related with those of the $m=1$ as well as $m=3 k$ sets because of the nonlinear coupling between the modes. Fluid motions develop in the polar as well as the equatorial region and the time dependence is similar to that of the $m=1$ set. The EA part rotates azimuthally (retrograde) without changing shape and is $\tau$ periodic whilst the ES part is prograde but changing shape and the period is $\tau / 2$.

We note that for the three sets of modes the observed change of shape is very weak or absent. From this analysis we conclude the functional form of a MRW in the MSC system is more complicated than that given in Eq. (8) (or Eq. (12)) of [4] in the context of Taylor-Couette flow. In particular, time dependence is not only associated to the $\varphi$ (azimuthal) coordinate as in [4, but also to the radial and colatitudinal coordinates as is reflected by the change of shape of the ES part of the flow (Table 3). However, their functional form remains valid in the case of the EA part of the flow because no shape change is observed in the rotating frame and the patterns are only azimuthally drifting. In addition, 
because the EA part of the flow is larger than the ES part and the shape changes associated with this part are quite weak (see supplementary movies), its volume averaged properties exhibit very small modulations as reproduced in the time series of Fig. 4(b,c).

\section{2 $\quad \mathrm{MRW}_{2}^{2}$ at $\mathrm{Ha}=5$}

The three sets of modes for the $\mathrm{MRW}_{2}^{2}$ at $\mathrm{Ha}=5$ are the most energetic nonaxisymmetric modes $m=2, m=4$ and $m=6$. Their kinetic energy ratios are $K_{m=2} / K_{m>0}=0.86, K_{m=4} / K_{m>0}=0.10$ and $K_{m=6} / K_{m>0}=0.03$. The outcomes of the mode analysis for the $\mathrm{MRW}_{2}^{2}$ are presented in Fig. 7 (three bottom rows) and Table 3 (also supplementary movies). The results are different for the case of $\mathrm{MRW}_{1}^{3}$. Now the time evolution (recall Eq. (8)) for the three sets, their ES as well as EA part, exhibit a change of the pattern while it is drifting or steady in the azimuthal direction. The significant change of shape of the modes results in noticeable oscillations of the volume averaged properties (see Fig. 3(b) at $\mathrm{Ha}=5$ on the $\mathrm{MRW}_{2}^{2}$ branch). As for the $\mathrm{MRW}_{1}^{3}$ the ES and EA part of each mode have different periods $\tau / 2$ and $\tau$. We note that the azimuthal drift is always prograde but develops in a nonuniform fashion and that the dominant azimuthal wave number of the EA part of the flow is $m=2$ while that of the ES part is doubled as is the case for the $\mathrm{MRW}_{1}^{3}$. Although the sets of modes (columns in Fig. 7) have the position of the maximum nonaxisymmetric radial velocity in the equatorial region, polar fluid motions are noticeable in the ES $m=2$ as well as EA $m=4$ modes (also slightly the $m=6$ ES mode, see meridional sections). The time dependence of the EA (ES) component of the $m=4$ mode is qualitatively similar to the ES (EA) component of the $m=2,6$ modes, exhibiting a stronger change of shape. This may be an indication that these flow components are strongly correlated with the corresponding Floquet mode giving rise to the $\mathrm{MRW}_{2}^{2}$ branch.

\section{3 $\mathrm{MRW}_{1}^{2}$ at $\mathrm{Ha}=5$ : branch 1 and 2}

Finally, the sets for the $\mathrm{MRW}_{1}^{2}$ on branch 1 and 2 at $\mathrm{Ha}=5$ are the modes $m=$ $1, m=2 k$ and $m=3$ which have $K_{m=1} / K_{m>0}=0.007, K_{m=2 k} / K_{m>0}=0.94$ and $K_{m=3} / K_{m>0}=0.032$ in the case of branch 1 and $K_{m=1} / K_{m>0}=0.017$, $K_{m=2 k} / K_{m>0}=0.87$ and $K_{m=3} / K_{m>0}=0.080$ for the $\mathrm{MRW}_{1}^{2}$ on branch 2. The time evolution of all the sets of modes in both branches is very similar. The patterns for each set are shown in the columns of Fig. 8 and their type of time dependence is outlined in Table 3. As happened for the other MRW the dominant azimuthal wave number of the EA part of the flow is $m=2$ while that of the ES part is doubled to $m=4$. In addition, noticeable polar fluid motions are present in the $m=1$ mode and the $m=2 k$ mode does not exhibit any azimuthal drift. This gives more support for the hypothesis that $M_{R} W_{1}^{2}$ bifurcate from a RW branch with $m=2$ azimuthal symmetry and that their Floquet mode has azimuthal symmetry $m=1$. Moreover, the most noticeable difference between the $\mathrm{MRW}_{1}^{2}$ on both branches is seen in the $m=1$ and $m=3$ 
Table 3: Summary of the time evolution (in the frame of reference rotating with frequency $\omega$ ) of each set of dominant modes for the $\mathrm{MRW}_{1}^{3}$ at $\mathrm{Ha}=3.5$, the $\mathrm{MRW}_{2}^{2}$ at $\mathrm{Ha}=5$, and the $\mathrm{MRW}_{1}^{2}$ on branch 1 and 2 , both at $\mathrm{Ha}=5$. In all the modes the observed change of shape is very weak or absent and the pattern is azimuthally rotating or fixed. In some cases (marked with *) the azimuthal drift is nonuniform. Polar or equatorial position of the maximum of the radial velocity of each mode is also stated. The time evolution of each mode (each row in the table) over a period $\tau$ is displayed in the supplementary movies for the $\mathrm{MRW}_{1}^{3}$ and $\mathrm{MRW}_{2}^{2}$.

$$
\mathrm{MRW}_{1}^{3} \text { at } \mathrm{Ha}=3.5
$$

\begin{tabular}{lllllll}
\hline$m$ & Eq. Sym. & $\max v_{r}$ & period & drift & shape change & Position max $v_{r}$ \\
\hline 1 & -1 & 0.011 & $\tau / 2$ & retrograde & 0 & Polar \\
1 & 1 & 0.018 & $\tau$ & prograde & 1 (weak) & Polar \\
2 & -1 & 0.015 & $\tau$ & retrograde & 0 & Equatorial \\
2 & 1 & 0.0025 & $\tau / 2$ & prograde & 1 & Polar \\
$3 k$ & -1 & 0.085 & - & none & 0 & Equatorial \\
$3 k$ & 1 & 0.041 & $\tau / 2$ & none & 1 & Equatorial \\
\hline \multicolumn{5}{c}{ MRW $_{2}^{2}$ at Ha $=5$} \\
\\
\hline$m$ & Eq. Sym. & \multirow{2}{*}{$\max v_{r}$} & period & drift & shape change & Position max $v_{r}$ \\
\hline 2 & -1 & 0.11 & $\tau / 2$ & none & 1 (weak) & Equatorial \\
2 & 1 & 0.0026 & $\tau$ & prograde* & 1 & Equatorial \\
4 & -1 & 0.0014 & $\tau$ & prograde* & 1 & Equatorial \\
4 & 1 & 0.052 & $\tau / 2$ & none & 1 (weak) & Equatorial \\
6 & -1 & 0.017 & $\tau / 2$ & none & 1 & Equatorial \\
6 & 1 & 0.0014 & $\tau$ & prograde* & 1 & Equatorial \\
\hline
\end{tabular}

$\mathrm{MRW}_{1}^{2}$ (branch 1) at $\mathrm{Ha}=5$

\begin{tabular}{|c|c|c|c|c|c|c|}
\hline$m$ & Eq. Sym. & $\max v_{r}$ & period & drift & shape change & Position $\max v_{r}$ \\
\hline 1 & -1 & 0.0048 & $\tau$ & prograde* & 1 & Equatorial \\
\hline 1 & 1 & 0.0089 & $\tau$ & retrograde* & 1 (weak) & Equatorial \\
\hline $2 k$ & -1 & 0.094 & $\tau / 2$ & none & 1 & Equatorial \\
\hline $2 k$ & 1 & 0.054 & $\tau / 2$ & none & 1 & Equatorial \\
\hline 3 & -1 & 0.0075 & $\tau$ & retrograde* & 1 & Equatorial \\
\hline 3 & 1 & 0.0087 & $\tau$ & prograde* & 1 & Equatorial \\
\hline \multicolumn{7}{|c|}{$\mathrm{MRW}_{1}^{2}($ branch 2$)$ at $\mathrm{Ha}=5$} \\
\hline$m$ & Eq. Sym. & $\max v_{r}$ & period & drift & shape change & Position max $v_{r}$ \\
\hline 1 & -1 & 0.0033 & $\tau$ & prograde* & 1 & Equatorial \\
\hline 1 & 1 & 0.017 & $\tau$ & retrograde* & 1 (weak) & Equatorial \\
\hline $2 k$ & -1 & 0.093 & $\tau / 2$ & none & 1 & Equatorial \\
\hline $2 k$ & 1 & 0.05 & $\tau / 2$ & none & 1 & Equatorial \\
\hline 3 & -1 & 0.015 & $\tau$ & retrograde* & 1 (weak) & Equatorial \\
\hline 3 & 1 & 0.0091 & $\tau$ & prograde* & 1 & Equatorial \\
\hline
\end{tabular}



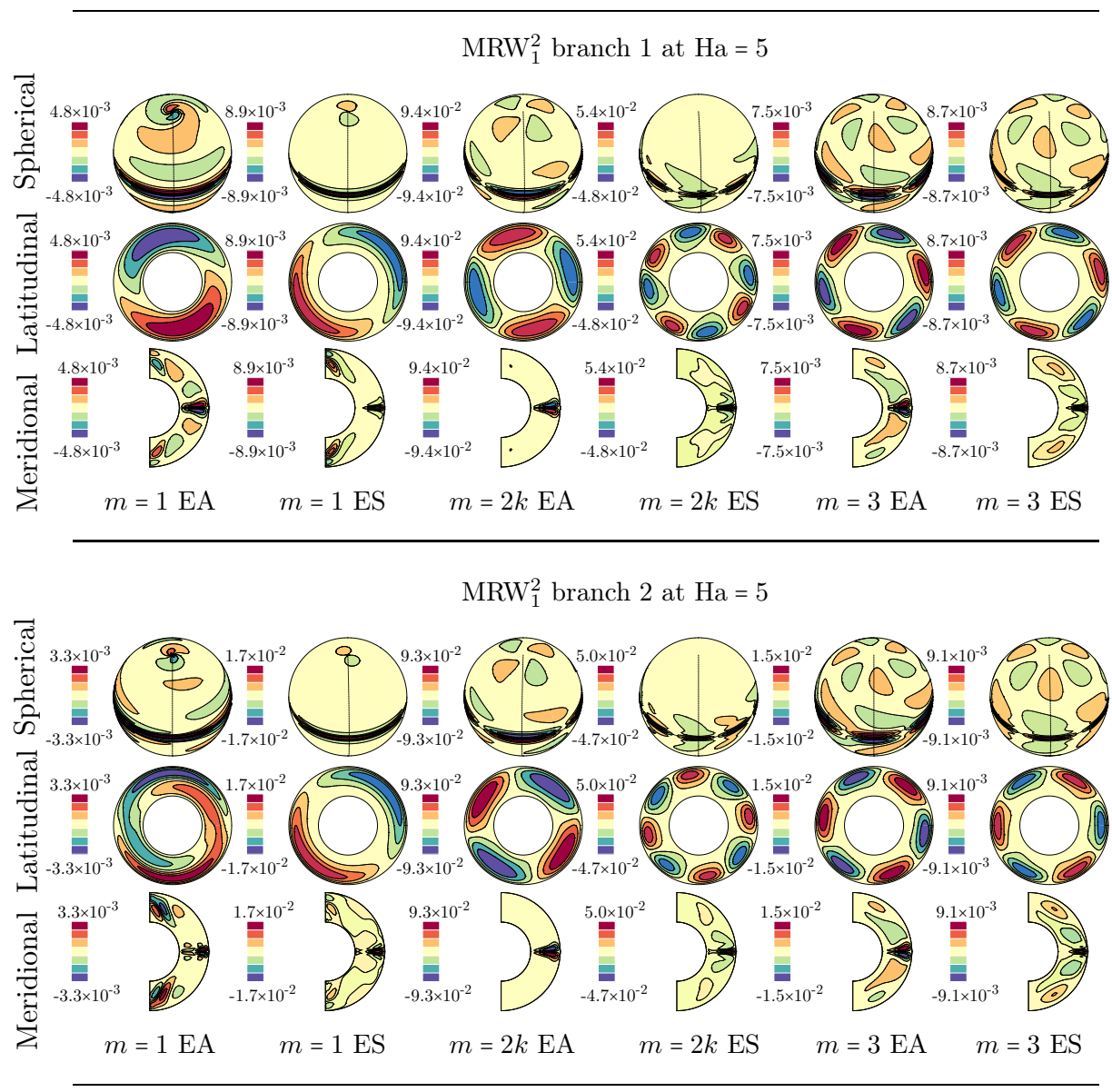

Figure 8: Most energetic $m=1,2 k, 3$ equatorially antisymmetric (EA) or symmetric modes (ES) of the $\mathrm{MRW}_{1}^{2}$ at $\mathrm{Ha}=5$ on branch 1 (top 3 rows) and of the $\mathrm{MRW}_{1}^{2}$ on branch 2 at $\mathrm{Ha}=5$ (bottom 3 rows). Cross-sections are as Fig. 6 but the spherical section point of view is at $45^{\circ}$ latitude. 
mode while the $m=2 k$ modes behave quite similarly ( $\omega$ and $\tau$ are quite similar as well, see Table. 22. This may be an indication that both branches bifurcate at different points of the same branch of RW with azimuthal symmetry $m=2$. To fully understand the origin of the two branches of $\mathrm{MRW}_{1}^{2}$ a continuation method for MRW as in [12] must be used.

\section{Summary}

Modulated rotating waves (MRW) in the magnetized shperical Couette system were computed for the radial jet instability [19] and few for the return flow [21] instability. The former for Hartmann numbers $\mathrm{Ha}<10$ and the later very close to $\mathrm{Ha}=30$, both for fixed Reynolds number $\mathrm{Re}=10^{3}$. These values correspond to experimental runs of the HEDGEHOG experiment already obtained [27] and fall in the interval where branches of rotating waves (RW) with $m=2,3,4$-fold azimuthal symmetry have been recently obtained [14. MRW are obtained by means of direct numerical simulations (DNS) from initial conditions given by the stability (Floquet) analysis of RW performed in [14] which provides estimations for the time scales and symmetries of bifurcated MRW. The main findings of the present study are summarized in the following.

For the equatorially asymmetric radial jet instability $(\mathrm{Ha} \sim 5)$ several types of MRW, and even 3 frequency time dependent flows, are described and their regions of stability determined. Several large intervals of multistability of MRW are found. This parameter regime is a good candidate for searching MRW in the HEDGEHOG experiment. In contrast, in case of the equatorially symmetric return flow instability $(\mathrm{Ha} \sim 30)$ MRW are very rare and their regions of stability very narrow. Almost only rotating waves (RW) described in [14 can be found because of the moderate value of Re. This parameter regime is then a good candidate for searching RW in the HEDGEHOG experiment. Broader stability intervals for MRW corresponding to the return flow instability may appear with increasing Re as shown in [19] for the aspect ratio $\chi=0.33$.

From the RW with three-fold azimuthal symmetry, bifurcated from the base axisymmetric state at $\mathrm{Ha}_{\mathrm{c}}=12.2$, the typical Ruelle-Takens scenario, giving rise to $m=1$-fold azimuthal symmetric MRW and 3 frequency radial jet time dependent flows, has been found by decreasing the Hartmann number. The flows belonging to this scenario have their kinetic energy mostly contained in the $m_{\max }=3$ azimuthal wave number matching the symmetry of the parent RW. However, these MRW do not exhibit noticeable oscillations of volume averaged physical properties, as is commonly found [19, 13], which may lead a wrong classification as RW if only volume-averaged properties are investigated.

We follow the theoretical studies of [33] and [4] and provide a description of the different types of MRW found in terms of their classification. In particular, the spatio-temporal symmetries of the MRW [33] and their connection with RW and Floquet modes [4] are rigorously stated. The figures and supplementary movies along a modulation period for a representative set of MRW provide an easy visualisation of the theoretical classification. 
By investigating the evolution of the most energetic azimuthal wave numbers in the spherical harmonics expansion of a MRW, a careful analysis of the spatiotemporal symmetries of the different azimuthal modes (equatorially symmetric or antisymmetric) for a selected types of MRW corresponding to the radial jet instability is performed. The study of MRW in terms of its dominant modes with $m=1,2,3$-fold fixed azimuthal symmetry allows us to determine the regions (equatorial or polar) within the shell with maximum signal (i.e. large flow velocities) for each class of azimuthal symmetry in order to constrain the measurement set-up in the HEDGEHOG experiment.

A characteristic difference between MRW and their corresponding parent RW is that the onset of modulation is associated with the appearance of noticeable flow velocities in the polar regions. A careful inspection of the equatorial symmetry evidences that the dominant azimuthal wave number of the flow $m_{\max }$

corresponds to that of the antisymmetric flow whilst it is doubled in the case of symmetric flow. This is relevant for future runs of the HEDGEHOG experiment as any measurement in the equatorial plane refers to the equatorially symmetric part of the flow.

Finally, we give numerical evidence that the time evolution of a specific single mode $m$, viewed in the frame azimuthally drifting with the corresponding frequency, can not only be associated with the azimuthal coordinate as in 4], hence a time dependence on the radial as well colatitudinal coordinates needs to be considered. MRW with nearly constant mean properties can be accurately approximated by nonlinear interaction of their dominant modes, which are either almost steadily azimuthally rotating or fixed exhibiting a weak change of the flow topology as the time dependence of their volume-averaged properties suggests. MRW with oscillating mean properties have some dominant modes, which are changing their pattern while also are azimuthally rotating or fixed. For these MRW the azimuthal drift seen in some azimuthal modes takes place in nonuniform fashion.

\section{Acknowledgements}

F. Garcia kindly acknowledges the Alexander von Humboldt Foundation for its financial support.

\section{References}

[1] Abbas, S., Yuan, L., Shah, A.: Simulation of spiral instabilities in wide-gap spherical Couette flow. Fluid Dyn. Res. 50(2), 025,507 (2018)

[2] Balbus, S.A., Hawley, J.F.: A powerful local shear instability in weakly magnetized disks. i- Linear analysis. ii- Nonlinear evolution. Astrophys. J. 376, 214-233 (1991) 
[3] Brito, D., Alboussière, T., Cardin, P., Gagnière, N., Jault, D., La Rizza, P., Masson, J.P., Nataf, H.C., Schmitt, D.: Zonal shear and super-rotation in a magnetized spherical Couette-flow experiment. Phys. Rev. E 83, 066,310 (2011)

[4] Coughlin, K.T., Marcus, P.S.: Modulated waves in Taylor-Couette flow. Part 1. Analysis. J. Fluid Mech. 234, 1-18 (1992)

[5] Crawford, J.D., Knobloch, E.: Symmetry and symmetry-breaking bifurcations in fluid dynamics. Ann. Rev. Fluid Mech. 23(1), 341-387 (1991)

[6] Dormy, E., Soward, A.M. (eds.): Mathematical Aspects of Natural Dynamos, The Fluid Mechanics of Astrophysics and Geophysics, vol. 13. Hartman \& Hall/CRC (2007)

[7] Ecke, R.E., Zhong, F., Knobloch, E.: Hopf bifurcation with broken reflection symmetry in rotating Rayleigh-Bénard convection. Europhys. Lett. 19, 177-182 (1992)

[8] Eckmann, J.P.: Roads to turbulence in dissipative dynamical systems. Rev. Modern Phys. 53(4), 643-654 (1981)

[9] Figueroa, A., Schaeffer, N., Nataf, H.C., Schmitt, D.: Modes and instabilities in magnetized spherical Couette flow. J. Fluid Mech. 716, 445-469 (2013)

[10] Frigo, M., Johnson, S.G.: The design and implementation of FFTW3. Proceedings of the IEEE 93(2), 216-231 (2005). Special issue on "Program Generation, Optimization, and Platform Adaptation"

[11] Garcia, F., Net, M., García-Archilla, B., Sánchez, J.: A comparison of highorder time integrators for thermal convection in rotating spherical shells. J. Comput. Phys. 229, 7997-8010 (2010)

[12] Garcia, F., Net, M., Sánchez, J.: Continuation and stability of convective modulated rotating waves in spherical shells. Phys. Rev. E 93, 013,119 (2016)

[13] Garcia, F., Sánchez, J., Dormy, E., Net, M.: Oscillatory convection in rotating spherical shells: Low Prandtl number and non-slip boundary conditions. SIAM J. Appl. Dynam. Systems 14(4), 1787-1807 (2015)

[14] Garcia, F., Stefani, F.: Continuation and stability of rotating waves in the magnetized spherical couette system: Secondary transitions and multistability. Proc. Roy. Soc. Lond. A 474, 20180,281 (2018)

[15] Gissinger, C., Ji, H., Goodman, J.: Instabilities in magnetized spherical Couette flow. Phys. Rev. E 84, 026,308 (2011) 
[16] Golubitsky, M., LeBlanc, V.G., Melbourne, I.: Hopf bifurcation from rotating waves and patterns in physical space. J. Nonlinear Sci. 10, 69-101 (2000)

[17] Golubitsky, M., Stewart, I.: The symmetry perspective: From equilibrium to chaos in phase space and physical space. Birkhäuser, Basel (2003)

[18] Goto, K., van de Geijn, R.A.: Anatomy of high-performance matrix multiplication. ACM Trans. Math. Softw. 34(3), 1-25 (2008)

[19] Hollerbach, R.: Non-axisymmetric instabilities in magnetic spherical Couette flow. Proc. Roy. Soc. Lond. A 465, 2003-2013 (2009)

[20] Hollerbach, R., Junk, M., Egbers, C.: Non-axisymmetric instabilities in basic state spherical Couette flow. Fluid Dyn. Res. 38, 257-273 (2006)

[21] Hollerbach, R., Skinner, S.: Instabilities of magnetically induced shear layers and jets. Proc. Roy. Soc. Lond. A 457, 785-802 (2001)

[22] Ji, H., Balbus, S.: Angular momentum transport in astrophysics and in the lab. Phys. Today 66(8), 27-33 (2013)

[23] Jones, C.A.: Planetary magnetic fields and fluid dynamos. Ann. Rev. Astron. Astrophys. 43(1), 583-614 (2011)

[24] Jordan, D., Smith, P.: Nonlinear Ordinary Differential Equations : An Introduction for Scientists and Engineers, Oxford Texts in Applied and Engineering Mathematics, vol. 10. Oxford University Press (2007)

[25] Kaplan, E.J.: Saturation of nonaxisymmetric instabilities of magnetized spherical Couette flow. Phys. Rev. E 85(063016), 1-8 (2014)

[26] Kaplan, E.J., Nataf, H.C., Schaeffer, N.: Dynamic domains of the Derviche Tourneur sodium experiment: Simulations of a spherical magnetized Couette flow. Phys. Rev. Fluids 3, 034,608 (2018)

[27] Kasprzyk, C., Kaplan, E., Seilmayer, M., Stefani, F.: Transitions in a magnetized quasi-laminar spherical Couette flow. Magnetohydrodynamics $\mathbf{5 3}(2), 393-401(2017)$

[28] Krupa, M.: Bifurcations of relative equilibria. SIAM J. Math. Anal. 21(6), 1453-1486 (1990)

[29] Laskar, J.: Frequency analysis of a dynamical system. Celestial Mech. Dyn. Astr. 56, 191-196 (1993)

[30] Mamun, C.K., Tuckerman, L.S.: Asymmetry and Hopf bifurcation in spherical Couette flow. Phys. Fluids 7, 80-91 (1995)

[31] Morley, N.B., Burris, J., Cadwallader, L.C., Nornberg, M.D.: GaInSn usage in the research laboratory. Review of Scientific Instruments 79(5), 056,107 (2008) 
[32] Palacios, A.: Cycling chaos in one-dimensional coupled iterated maps. Int. J. Bifurcation and Chaos 12(8), 1859-1868 (2002)

[33] Rand, D.: Dynamics and symmetry. Predictions for modulated waves in rotating fluids. Arch. Ration. Mech. An. 79(1), 1-37 (1982)

[34] Rüdiger, G.: Differential Rotation and Stellar Convection: Sun and Solartype Stars. Fluid mechanics of astrophysics and geophysics. Gordon and Breach Science Publishers (1989)

[35] Ruelle, D., Takens, F.: On the nature of turbulence. Comm. in Math. Physics 20, 167 (1971)

[36] Saad, Y., Schultz, M.H.: GMRES: a generalized minimal residual algorithm for solving nonsymmetric linear systems. SIAM J. Sci. Stat. Comput. 7, 865-869 (1986)

[37] Sánchez, J., Garcia, F., Net, M.: Computation of azimuthal waves and their stability in thermal convection in rotating spherical shells with application to the study of a double-Hopf bifurcation. Phys. Rev. E 87, 033,014/ 1-11 (2013)

[38] Sánchez, J., Net, M.: Numerical continuation methods for large-scale dissipative dynamical systems. Eur. Phys. J. Spec. Top. 225, 2465-2486 (2016)

[39] Schmitt, D., Alboussière, T., Brito, D., Cardin, P., Gagnière, N., Jault, D., Nataf, H.C.: Rotating spherical Couette flow in a dipolar magnetic field: experimental study of magneto-inertial waves. J. Fluid Mech. 604, 175-197 (2008)

[40] Schrauf, G.: The first instability in spherical Taylor-Couette flow. J. Fluid Mech. 166, 287-303 (1986)

[41] Seilmayer, M., Galindo, V., Gerbeth, G., Gundrum, T., Stefani, F., Gellert, M., Rüdiger, G., Schultz, M., Hollerbach, R.: Experimental evidence for nonaxisymmetric magnetorotational instability in a rotating liquid metal exposed to an azimuthal magnetic field. Phys. Rev. Lett. 113, 024,505 (2014)

[42] Sisan, D.R., Mujica, N., Tillotson, W.A., Huang, Y.M., Dorland, W., Hassam, A.B., Antonsen, T.M., Lathrop, D.P.: Experimental observation and characterization of the magnetorotational instability. Phys. Rev. Lett. 93, $114,502(2004)$

[43] Stefani, F., Gerbeth, G., Gundrum, T., Hollerbach, R., Priede, J., Rüdiger, G., Szklarski, J.: Helical magnetorotational instability in a Taylor-Couette flow with strongly reduced Ekman pumping. Phys. Rev. E 80, 066,303 (2009) 
[44] Stefani, F., Gundrum, T., Gerbeth, G., Rüdiger, G., Schultz, M., Szklarski, J., Hollerbach, R.: Experimental evidence for magnetorotational instability in a Taylor-Couette flow under the influence of a helical magnetic field. Phys. Rev. Lett. 97, 184,502 (2006)

[45] Stewartson, K.: On almost rigid rotations. Part 2. J. Fluid Mech. 26, 131-144 (1966)

[46] Travnikov, V., Eckert, K., Odenbach, S.: Influence of an axial magnetic field on the stability of spherical Couette flows with different gap widths. Acta Mech. 219, 255-268 (2011)

[47] Tuckerman, L.S., Langham, J., Willis, A.: Order-of-Magnitude Speedup for Steady States and Traveling Waves via Stokes Preconditioning in Channelflow and Openpipeflow, pp. 3-31. Springer International Publishing (2019)

[48] Wei, X., Hollerbach, R.: Magnetic spherical Couette flow in linear combinations of axial and dipolar fields. Acta Mech. 215, 1-8 (2010)

[49] Wicht, J.: Flow instabilities in the wide-gap spherical Couette system. J. Fluid Mech. 738, 184-221 (2014)

[50] Wulf, P., Egbers, C., Rath, H.J.: Routes to chaos in wide-gap spherical Couette flow. Phys. Fluids 11(6), 1359-1372 (1999)

[51] Yuan, L.: Numerical investigation of wavy and spiral Taylor-Görtler vortices in medium spherical gaps. Phys. Fluids 24(12), 124,104 (2012)

[52] Zikanov, O.Y.: Symmetry-breaking bifurcations in spherical Couette flow. J. Fluid Mech. 310, 293-324 (1996) 